Fourth International Conference on Sustainable Construction Materials and Technologies http://www.claisse.info/Proceedings.htm

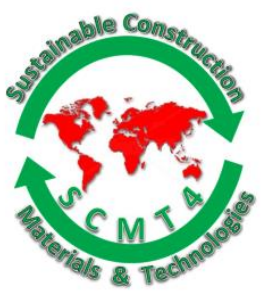

SCMT4

Las Vegas, USA, August 7-11, 2016

\title{
Feasibility Study of Municipal Solid Waste Incinerator Bottom Ash as Geopolymer Precursor
}

\author{
Weiping Zhu ${ }^{1 \mathrm{a}}$, Xu Chen ${ }^{2 \mathrm{a}}$, Leslie J. Struble ${ }^{2 \mathrm{~b}}$, and En-Hua Yang*1b \\ ${ }^{1}$ School of Civil and Environmental Engineering, Nanyang Technological University, Singapore \\ 639798.1aEmail:〈wzhu001@e.ntu.edu.sg>, ${ }^{1 b}$ Email:〈ehyang@ntu.edu.sg〉. \\ ${ }^{2}$ Civil and Environmental Engineering, Univ. of Illinois at Urbana-Champaign, Urbana, IL \\ 61801, United States of America. ${ }^{2 a}$ Email: <xuchen6@illinois.edu>, \\ ${ }^{2 b}$ Email: <lstruble@illinois.edu>.
}

\begin{abstract}
The potential of municipal solid waste incinerator bottom ash (IBA) as the sole precursor to synthesize geopolymer was investigated. Ground IBA powder was mixed with water glass and sodium hydroxide solution. After curing, the hardened samples were characterized by FTIR and NMR together with Salicylic acid/methanol extraction (SAM) to identify chemical composition of hardened binder. It was found that the IBA binder consists of one third of alkali-modified C-S-(A)-H and some geopolymeric gel with zeolite-like nano-structures.
\end{abstract}

\section{INTRODUCTION}

Geopolymer is a synthetic alkali-aluminosilicate binder formed through the reaction of solid aluminosilicate in highly concentrated aqueous alkaline solution (P. Duxson, 2007). It comprises of tetrahedral silicate and aluminate units linked in three-dimensional structure by covalent bonds, with negative charges associated with tetrahedral Al (III) charge-balanced by alkali cations. Starting materials essential for the formation of geopolymer consists of two main parts, the reactive solid aluminosilicate, and the alkali activator solution. Reactive solid aluminosilicate is typically derived from industrial by-product such as fly ash and alkali activator solution is commonly alkali metal hydroxide or silicate solution (Provis, 2014). Davidovits was the first to introduce the term 'geopolymer' in 1970s (Davidovits, 1979) but the technology of alkaliactivation was credited to Kuhl way back in 1908 (Kuehl, 1908). A major research was subsequently led by Glukovsky in 1950s (Glukhovsky, 1959), whereby he ventures into the commercial-scale production of geopolymer. From there onwards, geopolymer has gathered many interests from the academic world to conduct studies and research on the feasibility of producing a synthetic alkali aluminosilicate material for various types of applications. 
Incinerator ash is the by-product generated from incineration of municipal solid waste (MSW). Incineration of MSW has become global trend in waste management especially in land scarce cities as it mitigates the need of landfill. Incinerator bottom ash (IBA) accounts for $80 \%$ to $90 \%$ by weight of the total incinerator ash and is much less toxic. Typical compositions of IBA are silica, calcium, ferrous metal, iron oxide and aluminum oxide (Environmental Protection Agency, 2014). As waste disposal by incineration increases, there is need to develop innovative applications of IBA with environmental and economic benefits..

Currently, IBA is utilized as filler for road base construction (Santagata, et al, 2014), raw material for cement production ( $\mathrm{Li}$, et al, 2012), cement or aggregates substitute for concrete production (Kuo, et al, 2013), material for zeolites (Penilla, et al, 2003) and tobermorite synthesis (Jing, et al, 2007), and adsorbent for heavy metal removal (Zhang, et al, 2011). These approaches generally require complex and/or costly pre-treatments before it can be used for usually low value added applications. In this paper, the potential of IBA as the sole precursor to synthesize geopolymer was investigated. Ground IBA powder was mixed with water glass and sodium hydroxide solution. After curing, the hardened samples were characterized by FTIR and NMR to identify chemical composition of hardened binder.

\section{EXPERIMENTAL INVESTIGATION}

Raw materials. The IBA used in this research was collected from Keppel Seghers Tuas Waste to Energy Plant. IBA was first oven-dried at $105^{\circ} \mathrm{C}$ for 3 days and ground into fine particles with a rotating ball mill for 30 mins. Ground IBA was sieved and particles larger than $150 \mu \mathrm{m}$ were rejected. The chemical composition of the aforementioned IBA powder was determined by X-ray fluorescence (XRF), as summarized in Table 1. Similar to metakaolin, IBA contains high percentage of silicon and some aluminum, but it also has high calcium content, which may influence the reaction route of geopolymer as stated in Provis (2014).

Table 1. Chemical composition of IBA

\begin{tabular}{|l|c|c|c|c|c|c|c|c|c|c|c|}
\hline Formula & $\mathrm{SiO}_{2}$ & $\mathrm{Al}_{2} \mathrm{O}_{3}$ & $\mathrm{Na}_{2} \mathrm{O}$ & $\mathrm{K}_{2} \mathrm{O}$ & $\mathrm{TiO}_{2}$ & $\mathrm{Fe}_{2} \mathrm{O}_{3}$ & $\mathrm{CaO}$ & $\mathrm{MgO}$ & $\mathrm{P}_{2} \mathrm{O}_{5}$ & $\mathrm{SO}_{3}$ & $\mathrm{LOI}$ \\
\hline IBA (\%) & 32.75 & 8.57 & 2.87 & 1.24 & 1.57 & 10.02 & 29.06 & 1.75 & 4.77 & 3.01 & - \\
\hline
\end{tabular}

Sodium silicate solution (water glass) was supplied by International Scientific Pte Ltd with chemical composition: $\mathrm{Na}_{2} \mathrm{O} 9.65 \%, \mathrm{SiO}_{2} 29.16 \%$. Sodium hydroxide $(\mathrm{NaOH})$ pellets were provided by Schedelco Pte Ltd and has a purity of $99+\%$.

Experimental program. A bench top planetary mixer was used to mix ground IBA powder with alkaline activator: sodium hydroxide solution ( $8 \mathrm{M})$ and sodium silicate solution. The sodium hydroxide solution and the sodium silicate solution were mixed at a ratio of 1:2 by mass. The liquid to solid ratio of the resulting IBA paste was kept at 1.0. After mixing for 5 minutes, the fresh paste reached good consistency before it was cast into $50 \mathrm{~mm}$ cube moulds without vibration. The moulds were then sealed in plastic bags and cured in oven at temperature of $75{ }^{\circ} \mathrm{C}$ for three days. Samples were de-moulded after three days.

Chemical extraction, FTIR and NMR were used to study chemical composition of hardened binder. For the chemical extraction, salicylic acid dissolved in methanol (SAM) was used to remove calcium compounds including calcium silicates, calcium silicate hydrates, calcium hydroxides and calcium oxides (Gutteridge, 1979, Stutzman, 1996). For each $5 \mathrm{~g}$ of hardened IBA binder, 30g salicylic acid and 200mL methanol were used for extraction. 
Fourier transform infrared (FTIR) spectroscopy was used to characterize samples before and after SAM extraction. FTIR spectra of hardened IBA binder before SAM extraction (IBA binder), the residual of hardened IBA binder after SAM extraction (IBA binder SAM residual) and the soluble of hardened IBA binder after SAM extraction (IBA binder SAM soluble). Chemical bonds absorb particular wavelengths of infrared which can be identified in the Fourier transformed spectra. Therefore, absorption at various wavenumbers (a concept similar to frequency) corresponds to specific chemical bonds.

NMR is a powerful characterization technique to identify chemical structure of amorphous gel and has been used to analyse cement and geopolymer nano-structures. In this research, nuclei of ${ }^{29} \mathrm{Si}$ and ${ }^{27} \mathrm{Al}$ in IBA samples were tested by the Bruker Avance 400 (DRX400).

\section{RESULTS AND DISCUSSIONS}

The average strength of the samples after curing for three days is $2.8 \pm 0.3 \mathrm{MPa}$ with a density of $1.0 \pm 0.1$ $\mathrm{g} / \mathrm{cm}^{3}$ as shown in Figure 1.

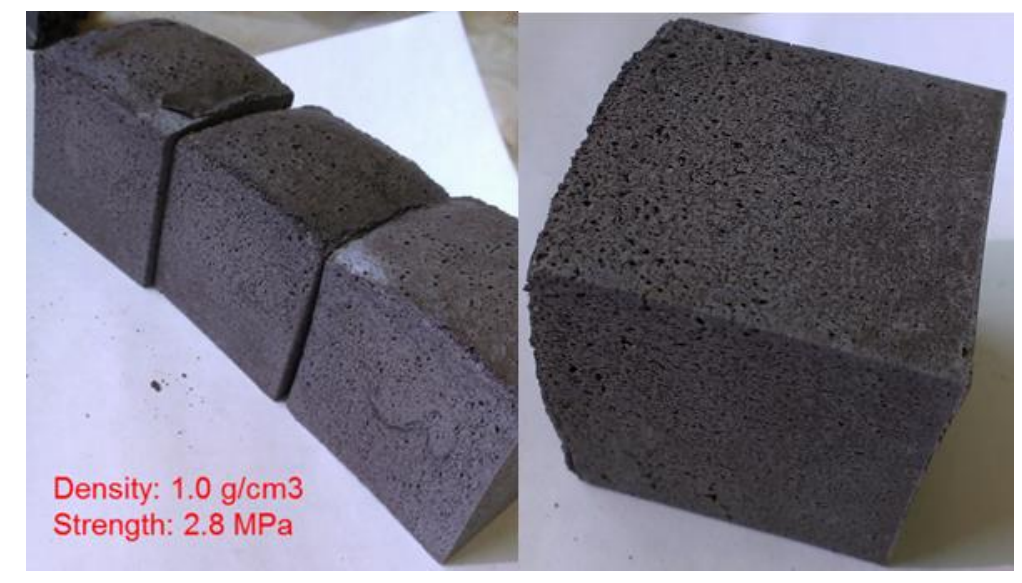

Figure 1. Hardened IBA binder cubes

Results of chemical extraction are summarized in Table 2. As can be seen, about $30 \%$ of calcium compounds were removed from the hardened IBA binder by SAM extraction.

Table. 2 SAM extraction on hardened IBA binder

\begin{tabular}{|c|c|c|}
\hline Before SAM & After SAM & Retaining (\%) \\
\hline $5 \mathrm{~g}$ & $3.644 \mathrm{~g}$ & $72.9 \%$ \\
\hline
\end{tabular}

FTIR-transmittance spectra of hardened IBA binder, residual of IBA binder after SAM and the soluble of IBA in SAM are show in Figure 2. The spectrum of IBA binder SAM soluble (dot dash line) was obtained by subtracting spectrum of IBA binder SAM residual (dash line) from spectrum of IBA binder (solid line), according to SAM results. It should be noted that because errors could arise during sample preparation for FTIR pellets and solids salvage for SAM extraction, spectrum obtained from subtraction would not be precisely correct and therefore should be considered semi-quantitative. 


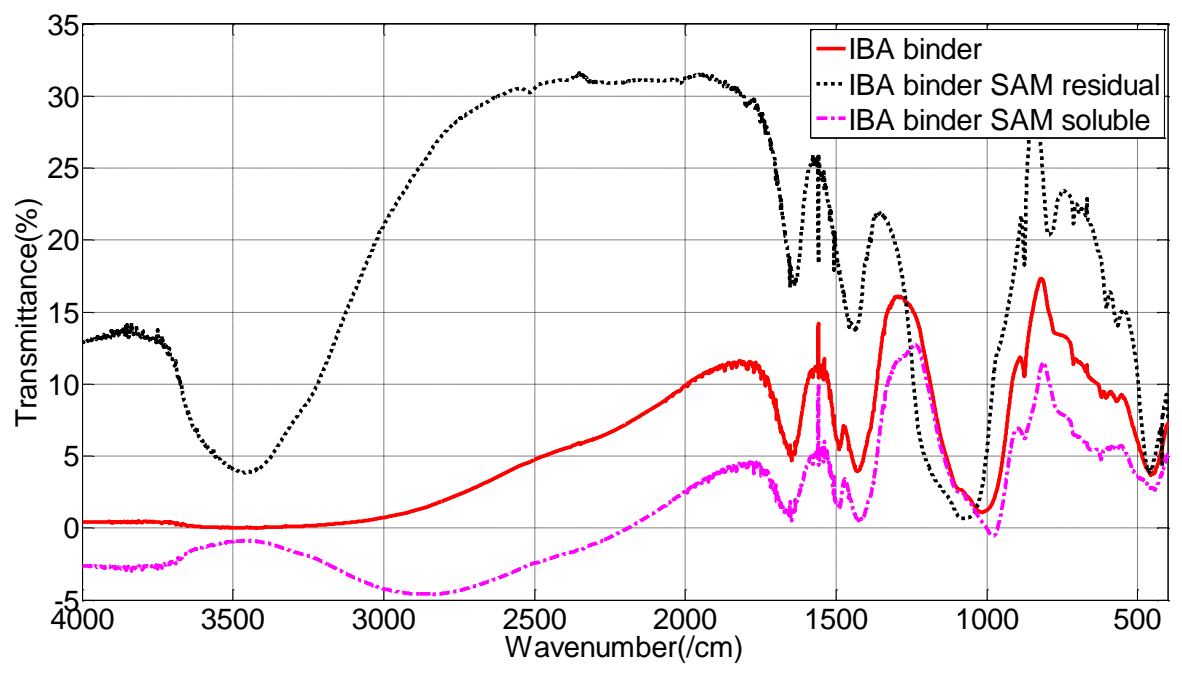

Figure 2. Full FTIR spectra of IBA binder, IBA binder SAM residual and IBA binder SAM soluble.

According to libraries from literature, wavenumbers between 850 and 1200 are associated to chemical structures of Si tetrahedral units. Figure 3 shows the FTIR spectra of IBA binder, IBA binder SAM residual and IBA binder SAM soluble in the wavenumber between 800 and 1,300.

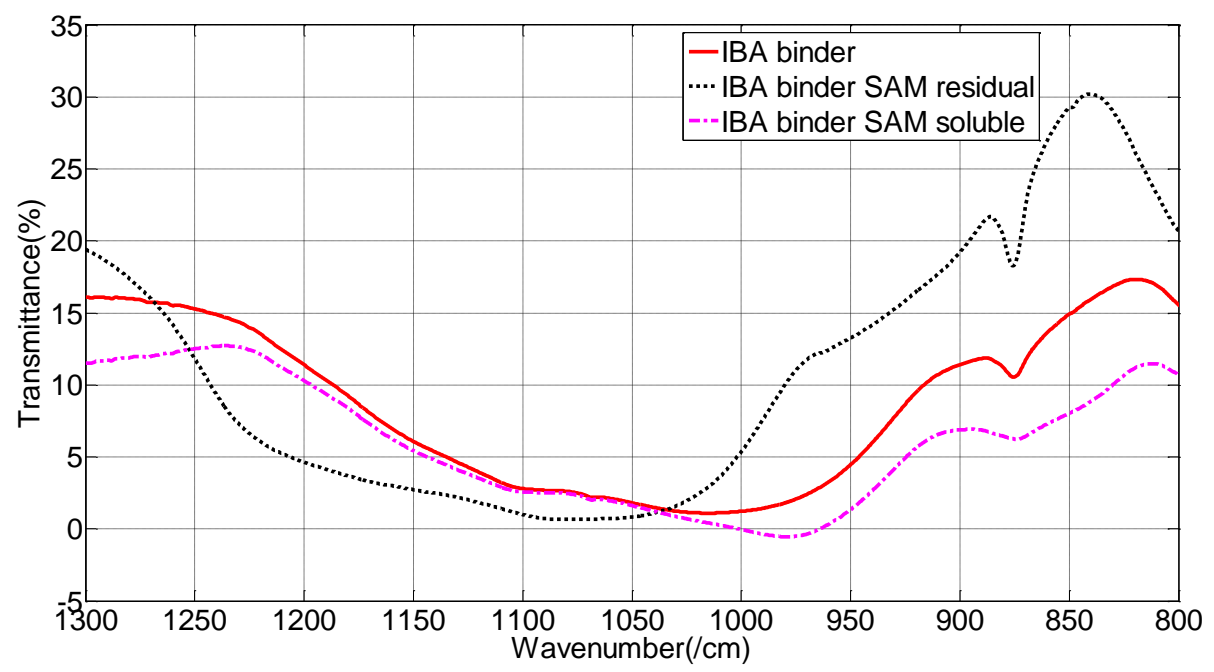

\section{Figure 3. FTIR spectra of IBA binder, IBA binder SAM residual and IBA binder SAM soluble in the wavenumber between 800 and 1,300}

As can be seen, the spectrum of the IBA binder SAM soluble (dot dash line) is different from the spectra of IBA binder (solid line) and IBA binder SAM residual (dash line). FTIR spectrum decomposition was carried out to identify hidden peaks due to spectrum overlapping. To decompose a spectrum, the FTIR signal was first taken second derivative to identify potential peaks. An arbitrary Gaussian function was then assigned to each peak. Superposition of all decomposed curves should be identical to the original spectrum. An iteration process was used to vary the amplitude and bandwidth of Gaussian function assigned to each peak until the accumulative curve fits well to the original spectrum. The decomposition of the IBA binder SAM soluble spectrum between 800 and 1200 is shown in Figure 4. 


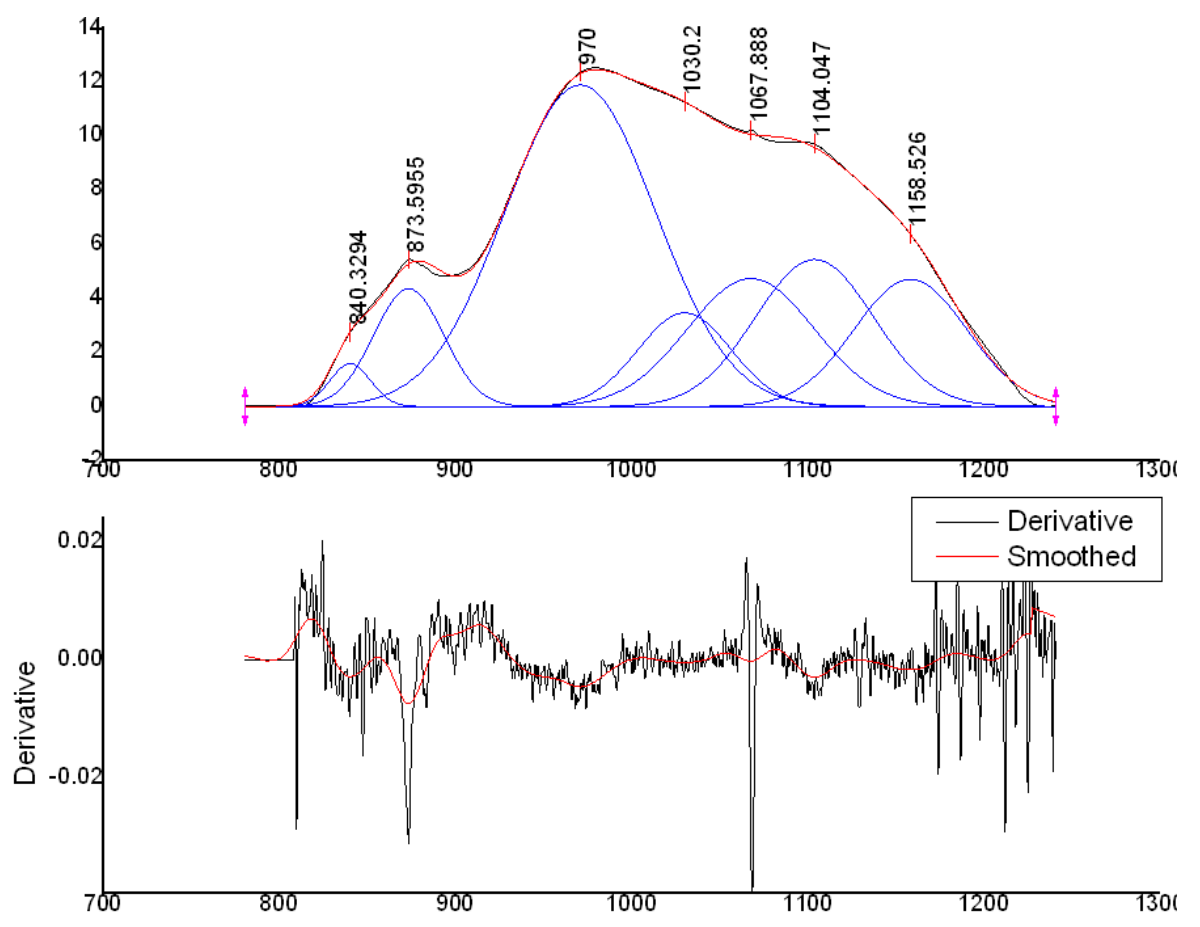

Figure 4. Decomposition of FTIR spectra of the SAM Soluble

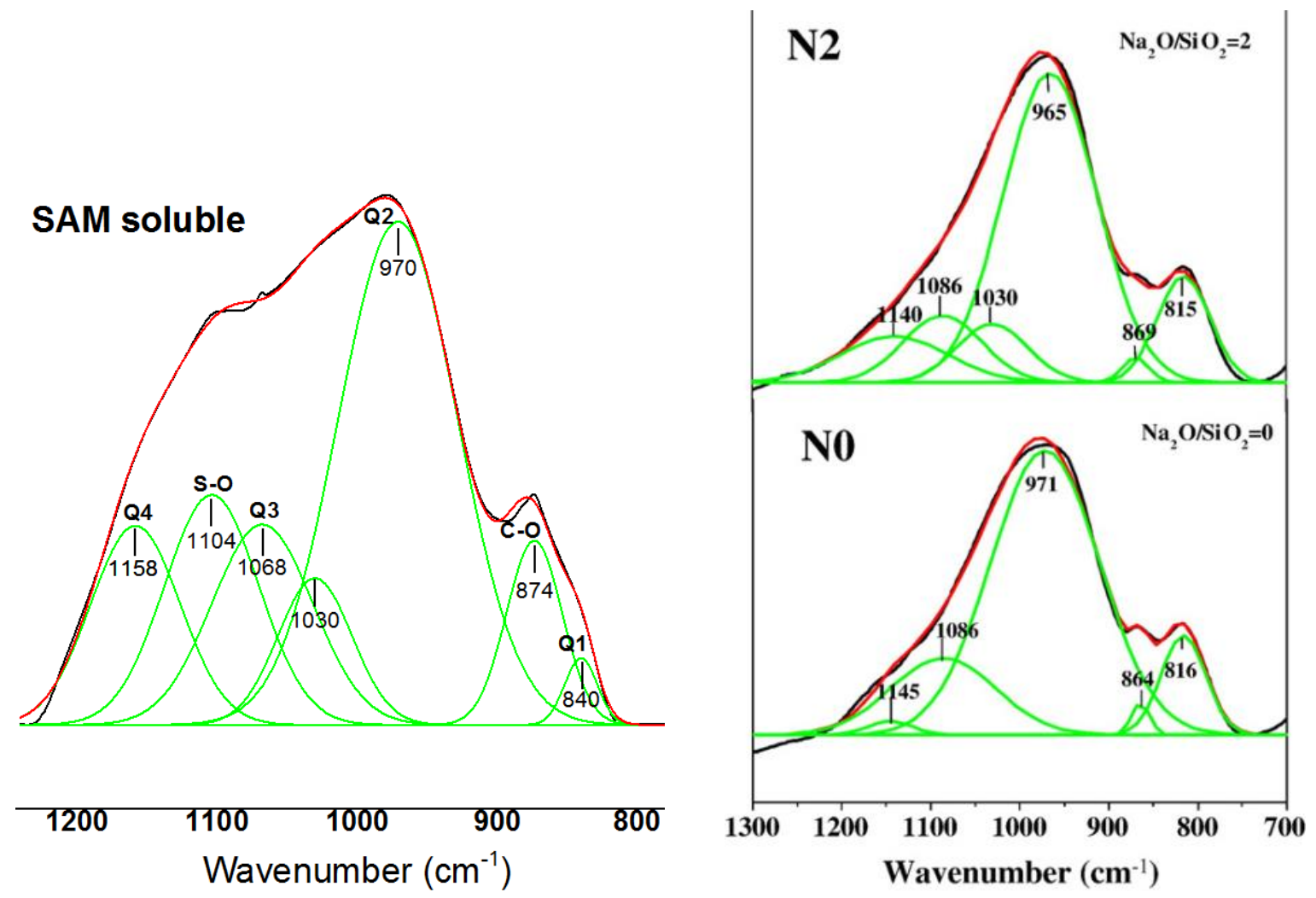

Figure 5. FTIR spectra of the SAM Soluble (left) and the literature results (Lodeiro, et al, 2009) 
Most decomposed components can be associated with Si-O-(Al) vibrations according to FTIR library from literature (Hanna, et al, 1995, Mollah, et al, 1998, Clayden, et al, 1999, Yu, et al, 1999), as denoted in Figure 5. Comparing with research results in literature (Lodeiro, et al, 2009) which dealt with effects of alkali on fresh $\mathrm{C}-\mathrm{S}-\mathrm{H}$ gel ( $\mathrm{N}$ denotes $\mathrm{Na} / \mathrm{Si}$ ratio, high $\mathrm{N}$ value indicates high alkalinity), the decomposition components here are very similar to that of C-S-H gel under high alkalinity, especially the one of N4. As can be seen from Figure 5, with alkali increase, in N2 and N4, a new peak around 1030 appeared, and the peak centered 815 migrated to 840 , which indicates C-S-H gel was modified by the alkali, leading to silicate polymerization (Lodeiro, et al, 2009). All the peaks in spectrum of N4 are also identified in spectrum of IBA binder SAM soluble, except for the peak of 1104 which was caused by S-O vibration, as IBA contains sulphates. The peak around 1150 is due to $\mathrm{Si}-\mathrm{O}$ vibration of Q4 with relative area percentage of $12.4 \%$. The fact that small amount of Q4 existed in SAM soluble suggests the insoluble residual after SAM was probably more than its true quantity and part of insoluble residual was accounted into the soluble; therefore, SAM extraction should be performed more carefully to improve data quality. Hence, it can be concluded that about $30 \%$ of IBA binder were probably alkali modified C-(A)-S-H gel.

To reveal chemical composition and structure of the remaining gel, FTIR decomposition of ground IBA powder, alkaline activator solution, and IBA binder SAM residual were carried out. As can be seen from the three spectra, three new peaks were found in the spectrum of IBA binder SAM residual which were not visible in spectrum of ground IBA powder and alkaline activator solution, as shown in Figure 6 and summarized in Table 3.
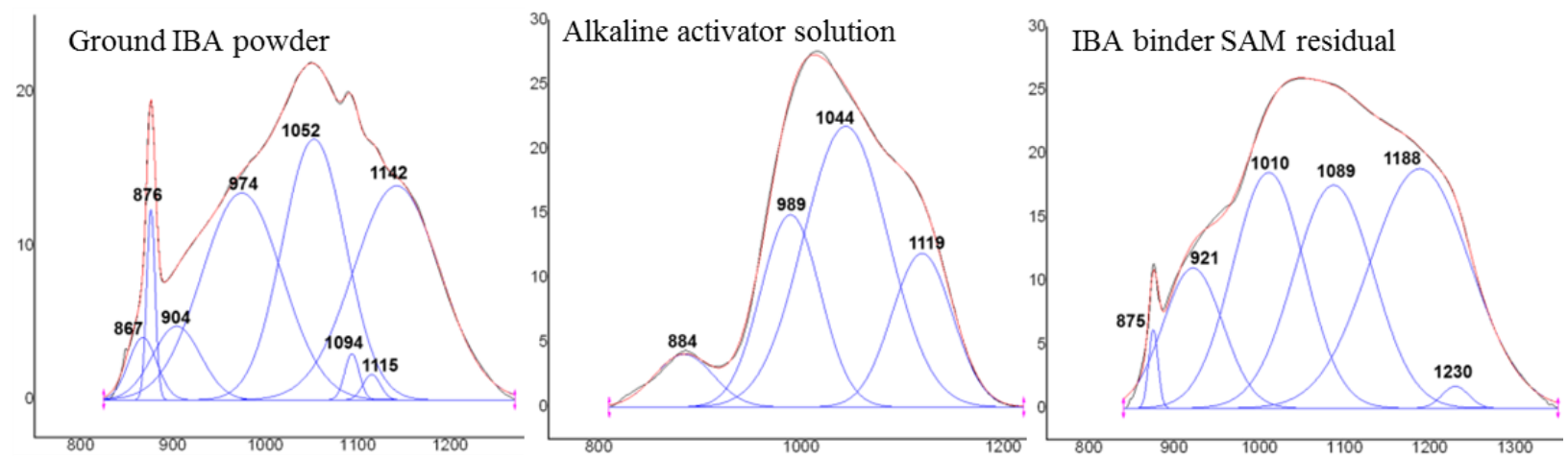

Figure 6. FTIR decomposition results of ground IBA powder, alkaline activator solution, and IBA binder SAM residual

Table 3. Peaks of FTIR spectra decomposition results of ground IBA powder, alkaline activator solution, and IBA binder SAM residual.

\begin{tabular}{|c|c|c|c|c|c|c|c|c|c|c|}
\hline Peak assigned & C-O & C-O & Si-O & Si-O & Si-O & Si-O & Si-O & S-O & Si-O & Si-O \\
\hline Ground IBA powder & 867 & 876 & & & 974 & & 1052 & 1094 & 1115 & \\
\hline Alkaline activator solution & & & 884 & & 989 & & 1044 & & 1119 & \\
\hline IBA binder SAM residual & & 875 & & 921 & & 1010 & & 1089 & & 1188 \\
\hline
\end{tabular}


The three new peaks found in IBA binder SAM residual are characteristic of Si-O-Si bridging bonds which are not identified in the precursor (ground IBA powder) and alkaline activator solution. This indicates the formation of zeolite-like chemical bonds due to reaction between IBA powder and alkaline activator. In summary, hardened IBA binder consists of a mixture of alkaline modified C-(A)-S-H gel as well as zeolitelike $\mathrm{Si}-\mathrm{O}-\mathrm{Si}(\mathrm{Al})$ geopolymer gel.

Because FTIR analysis results are usually considered semi-quantitative, NMR was carried out to further clarify quantitatively the degree of $\mathrm{Al}$ incorporation into the Si-O structure. The NMR spectra of ground IBA powder and hardened IBA binder are plotted in Figure 7.

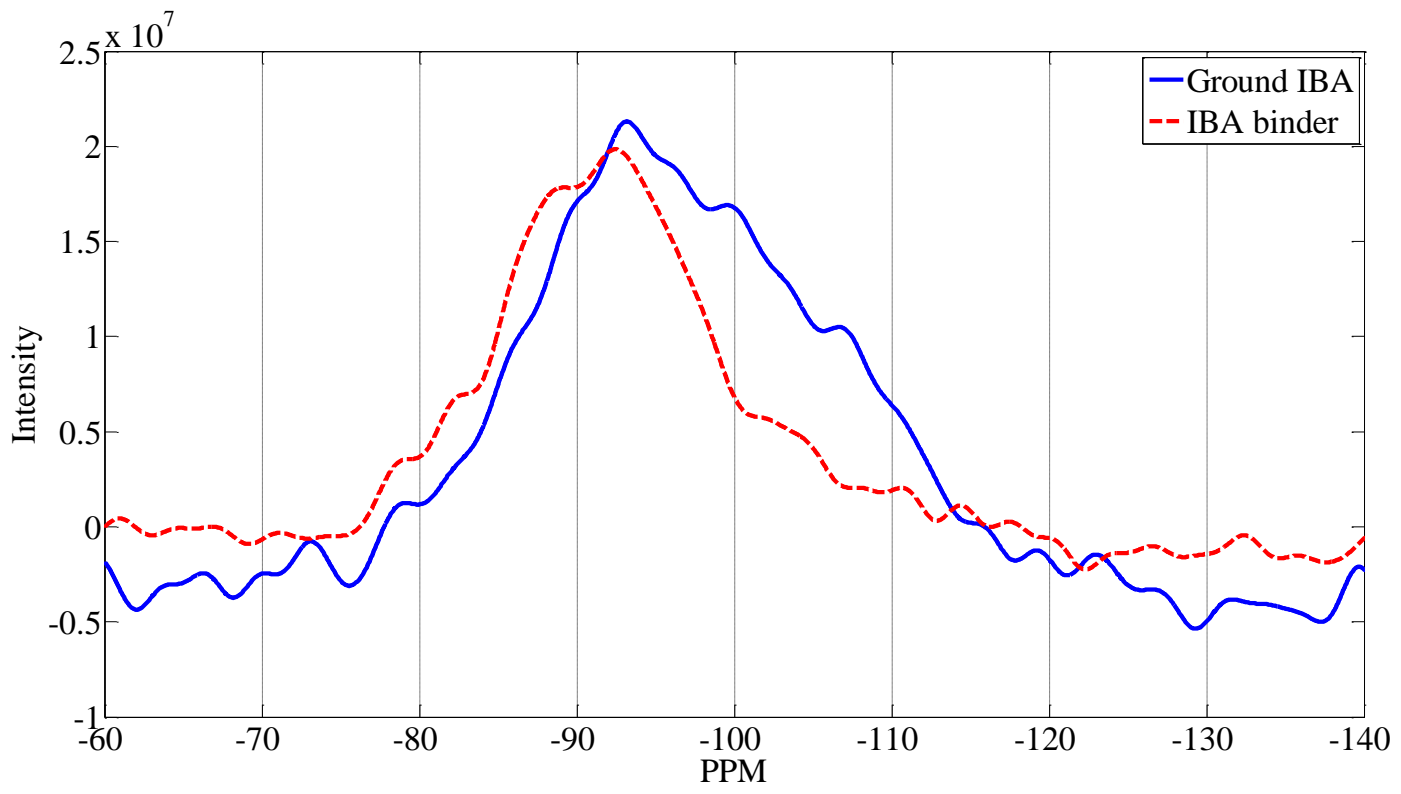

Figure 7. ${ }^{29} \mathrm{Si}$ NMR spectra of ground IBA powder and hardened IBA binder.

Broad peaks due to peaks overlapping were observed in Figure 7. NMR spectrum decomposition was carried out in a similar way to that of FTIR spectrum. Figure 8 shows decomposition results of ${ }^{29} \mathrm{Si}$ NMR spectra of ground IBA powder and hardened IBA binder.

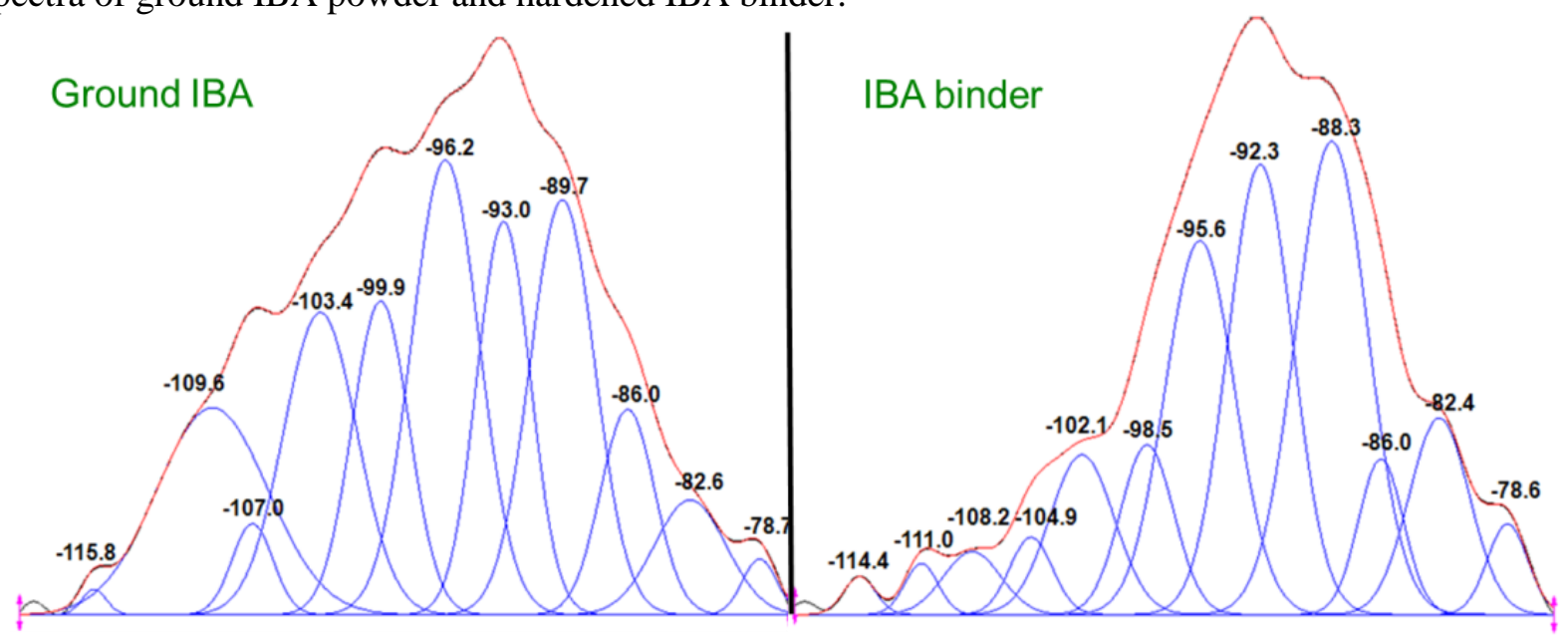

Figure 8. Decomposition of ${ }^{29}$ Si NMR spectra of ground IBA powder and hardened IBA binder 
As can be seen from Figure 8, most peaks in IBA spectrum are also present in the spectrum of hardened IBA binder with some changes in amplitude and area. This implies chemical bonds (chemical contents) had undergone some transformation, i.e parts of one group of chemical bonds reduced and another group of chemical bonds increased after thermal curing. To understand the participation of $\mathrm{Al}$ in hardened IBA binder, ${ }^{27} \mathrm{Al}$ NMR tests were conducted and the spectra are shown in Figure 9.

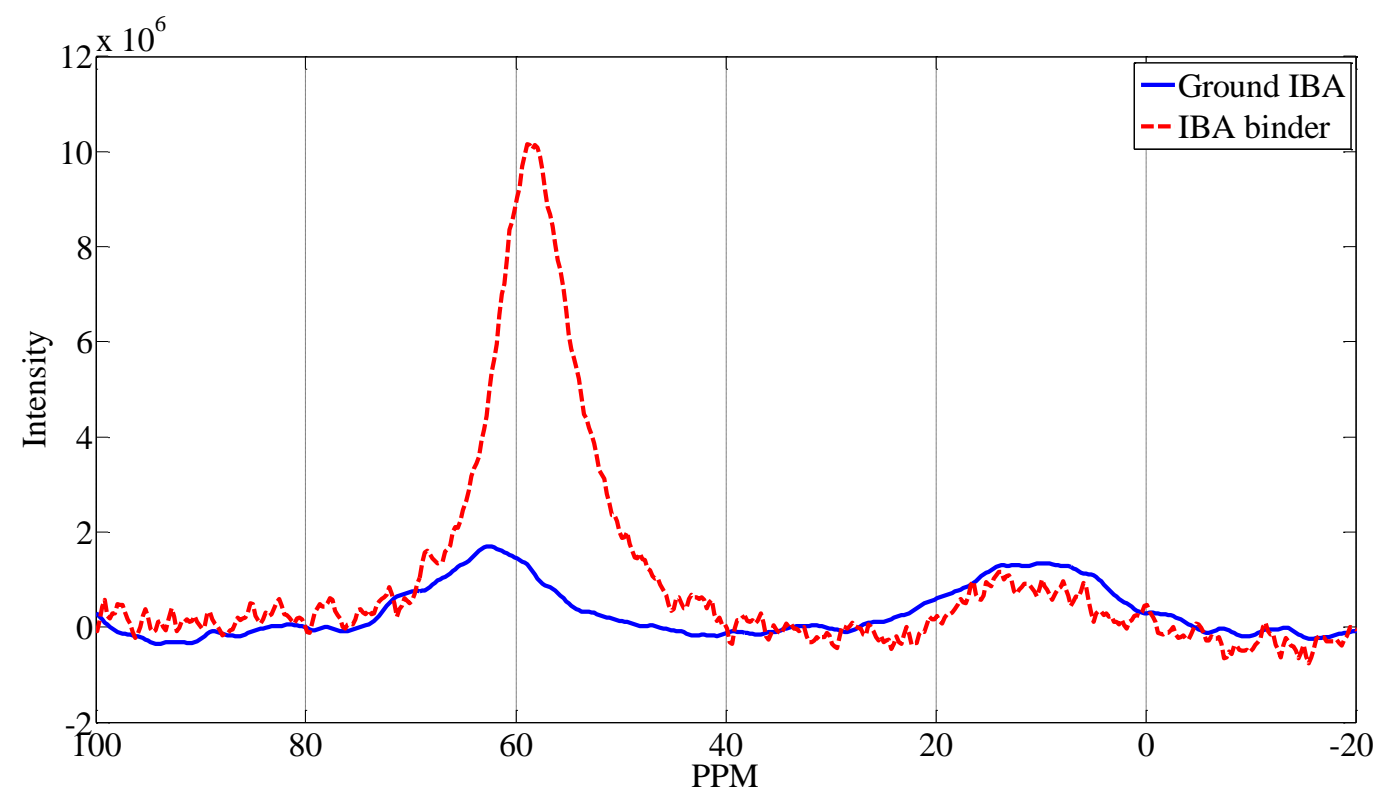

Figure 9. ${ }^{27} \mathrm{Al}$ NMR spectra of ground IBA powder and hardened IBA binder.

According to library from literature (Buchwald, et al, 2007), peaks around $60 \mathrm{ppm}$ denote tetrahedral coordination of $\mathrm{Al}$, while peaks around $0 \mathrm{ppm}$ suggest that $\mathrm{Al}$ is in the octahedral coordination. As can be seen in Figure 9, significant increase in the tetrahedral Al coordination (around 60 PPM) was found in hardened IBA binder. This suggests most of available aluminium had been incorporated into the $\mathrm{Si}-\mathrm{O}-\mathrm{Si}$ chain network. Hence, the percentage of $\mathrm{Q}(\mathrm{mAl})$ must have increased after the chemical reaction between ground IBA powder and alkaline activator. As a result, some peaks with relative area changed should be associated with $\mathrm{Q}(\mathrm{mAl})$, together with reference from peak assignments in comparative literature (Criado, Fernández-Jiménez et al. 2008), decomposition results of ${ }^{29} \mathrm{Si}$ NMR spectra are assigned with Q units in Table 4.

Table 4. Assignment of NMR decomposition results

\begin{tabular}{|c|c|c|c|c|c|c|c|c|c|}
\hline $\begin{array}{c}\text { Peak } \\
\text { assigned } \\
\text { area \%) }\end{array}$ & $\mathrm{Q}^{1}$ & $\mathrm{Q}^{2}(1 \mathrm{Al})$ & $\mathrm{Q}^{2}$ & $\mathrm{Q}^{4}(4 \mathrm{Al})$ & $\mathrm{Q}^{4}(3 \mathrm{Al})$ & $\mathrm{Q}^{3}$ & $\mathrm{Q}^{4}(2 \mathrm{Al})$ & $\mathrm{Q}^{4}(1 \mathrm{Al})$ & $\mathrm{Q}^{4}(1 \mathrm{Al})$ \\
\hline $\begin{array}{c}\text { Ground IBA } \\
\text { powder }\end{array}$ & -78.7 & -82.6 & -86.0 & -89.7 & -93.0 & -96.2 & -99.9 & -103.4 & \\
\hline IBA binder & -78.6 & $(4.9)$ & $(6.6)$ & $(16.1)$ & $(12.7)$ & $(17.9)$ & $(11.8)$ & $(12.9)$ & \\
\hline & $(2.58)$ & $(8.43)$ & $(4.97)$ & $(24.08)$ & $(20.77)$ & $(18.41)$ & $(6.44)$ & $(7.33)$ & $(2.34)$ \\
\hline
\end{tabular}

As can be seen from Table 4, numbers in the parenthesis are individual peak area percentages of the sum of all peak areas after decomposition, generally, $\mathrm{Q}^{2}(1 \mathrm{Al}), \mathrm{Q}^{4}(4 \mathrm{Al}), \mathrm{Q}^{4}(3 \mathrm{Al})$ increased greatly, while $\mathrm{Q}^{4}(0 \mathrm{Al})$, 
$\mathrm{Q}^{4}(1 \mathrm{Al})$ and $\mathrm{Q}^{4}(2 \mathrm{Al})$ sharply dropped, which are results of $\mathrm{Q}^{4}$ breakup and aluminium incorporation into Si-O chain network. Therefore, conclusions from NMR results are: (1) after thermal curing, most of aluminium available had become tetrahedral coordination form and been incorporated into Si-O chain network; (2) silicon-rich structural units $\left(\mathrm{Q}^{4}(2 \mathrm{Al}), \mathrm{Q}^{4}(1 \mathrm{Al})\right.$ and $\left.\mathrm{Q}^{4}(0 \mathrm{Al})\right)$ decreased, aluminium-rich structural units $\left(\mathrm{Q}^{4}(4 \mathrm{Al})\right.$ and $\left.\mathrm{Q}^{4}(3 \mathrm{Al})\right)$ increased, in other words, geopolymerization happened, as the essence of geopolymerization is the formation of $\mathrm{Si}-\mathrm{O}-\mathrm{Al}$ bonds and the increasing of $\mathrm{Q}^{4}(4 \mathrm{Al})$ and $\mathrm{Q}^{4}(3 \mathrm{Al})$; therefore, more 3-D silicon units connected with each other thanks to the bridging Al, since tetrahedral Al unites can only bridge rather than terminate a chain; (3) number of chain free ends also increased (increased $\left.\mathrm{Q}^{1}\right)$.

\section{CONCLUSION}

In this paper, the potential of IBA as the sole precursor to synthesize geopolymer was investigated. Ground IBA powder was first mixed with water glass and sodium hydroxide solution, and then was cured in oven at $60^{\circ} \mathrm{C}$ for three days. After curing, the hardened specimens were found with compressive strength around $3 \mathrm{MPa}$. FTIR and NMR identified its chemical composition of hardened binder, it consisted of alkalimodified C-S-(A)-H (about 30\%) and some geopolymeric gel which incorporated tedrehedral Al into its Si-O-Si network.

\section{ACKNOWLEDGEMENT}

The authors would like to acknowledge financial supports from the Environment Technology Research Programme (ETRP 1301 104), National Environment Agency of Singapore and SinBerBEST, National Research Foundation of Singapore.

\section{REFERENCES}

Buchwald, A., H. Hilbig and C. Kaps, (2007). "Alkali-activated metakaolin-slag blends-performance and structure in dependence of their composition.” Journal of materials science, 42(9): 3024-3032.

Clayden, N., S. Esposito, A. Aronne and P. Pernice, (1999). "Solid state 27 Al NMR and FTIR study of lanthanum aluminosilicate glasses." Journal of non-crystalline solids, 258(1): 11-19.

Davidovits, J. and S. Cordi, (1979). "Synthesis of new high-temperature geopolymers for reinforced plastics/composites." SPE PACTEC, 79: 151-154.

Duxson, P., A. Fernández-Jiménez, J. L. Provis, G. C. Lukey, A. Palomo and J. S. J. van Deventer. (2007). "Geopolymer technology: the current state of the art." Journal of Materials Science, 42(9): 2917-2933.

Environmental Protection Agency. (2014). "Ash Generated from the MSW Combustion Process." On: http://www.epa.gov/wastes/nonhaz/municipal/wte/basic.htm. [Accessed 1 February 2015].

Lodeiro, I. G., Macphee, D. E., Palomo, A., \& Fernández-Jiménez, A. (2009). “Effect of alkalis on fresh C-S-H gels. FTIR analysis." Cement and Concrete Research, 39(3), 147-153.

Glukhovsky, V. 1959). “Soil silicates (Gruntosilikaty).” USSR Kiev: Budivelnik Publisher.

Gutteridge, W. (1979). "On the dissolution of the interstitial phases in Portland cement." Cement and Concrete Research, 9(3): 319-324. 
Hanna, R., P. Barrie, C. Cheeseman, C. Hills, P. Buchler and R. Perry. (1995). "Solid state 29 Si and 27 Al NMR and FTIR study of cement pastes containing industrial wastes and organics." Cement and concrete research, 25(7): 1435-1444.

Jing, Z., F. Jin, N. Yamasaki and E. H. Ishida. (2007). "Hydrothermal synthesis of a novel tobermoritebased porous material from municipal incineration bottom ash." Industrial \& engineering chemistry research, 46(8): 2657-2660.

Kuehl, H. (1908). "Slag cement and process of making the same.” Google Patents.

Kuo, W.-T., C.-C. Liu and D.-S. Su. (2013). "Use of washed municipal solid waste incinerator bottom ash in pervious concrete." Cement and Concrete Composites, 37(0): 328-335.

Li, X.-G., Y. Lv, B.-G. Ma, Q.-B. Chen, X.-B. Yin and S.-W. Jian. (2012).” Utilization of municipal solid waste incineration bottom ash in blended cement.” Journal of Cleaner Production, 32(0): 96-100.

Mollah, M. Y. A., F. Lu and D. L. Cocke. (1998). "An X-ray diffraction (XRD) and Fourier transform infrared spectroscopic (FT-IR) characterization of the speciation of arsenic (V) in Portland cement typeV." Science of the total environment, 224(1): 57-68.

Penilla, R. P., A. G. Bustos and S. G. Elizalde. (2003). "Zeolite synthesized by alkaline hydrothermal treatment of bottom ash from combustion of municipal solid wastes." Journal of the American Ceramic Society, 86(9): 1527-1533.

Provis, J. L. (2014). “Geopolymers and other alkali activated materials: why, how, and what?" Materials and structures, 47(1-2): 11-25.

Stutzman, P. E. (1996). “Guide for X-ray powder diffraction analysis of Portland cement and clinker.” US Department of Commerce, Technology Administration, National Institute of Standards and Technology, Office of Applied Economics, Building and Fire Research Laboratory.

Yu, P., R. J. Kirkpatrick, B. Poe, P. F. McMillan and X. Cong. (1999). "Structure of calcium silicate hydrate (C-S-H): Near-, Mid-, and Far-infrared spectroscopy." Journal of the American Ceramic Society, 82(3): 742-748.

Zhang, H. Y., Y. Zheng, H. T. Hu and J. Y. Qi. (2011). "Use of municipal solid waste incineration bottom ash in adsorption of heavy metals.” Key Engineering Materials, 474: 1099-1102. 